\title{
The Role of Manganese Superoxide Dismutase in Skin Cancer
}

\author{
Delira Robbins and Yunfeng Zhao \\ Department of Pharmacology, Toxicology \& Neuroscience, Louisiana State University Health Sciences Center, \\ 1501 Kings Highway, Shreveport, LA 71130, USA \\ Correspondence should be addressed to Yunfeng Zhao, yzhao1@lsuhsc.edu
}

Received 14 September 2010; Accepted 26 January 2011

Academic Editor: Yong-Doo Park

Copyright ( 2011 D. Robbins and Y. Zhao. This is an open access article distributed under the Creative Commons Attribution License, which permits unrestricted use, distribution, and reproduction in any medium, provided the original work is properly cited.

\begin{abstract}
Recent studies have shown that antioxidant enzyme expression and activity are drastically reduced in most human skin diseases, leading to propagation of oxidative stress and continuous disease progression. However, antioxidants, an endogenous defense system against reactive oxygen species (ROS), can be induced by exogenous sources, resulting in protective effects against associated oxidative injury. Many studies have shown that the induction of antioxidants is an effective strategy to combat various disease states. In one approach, a SOD mimetic was applied topically to mouse skin in the two-stage skin carcinogenesis model. This method effectively reduced oxidative injury and proliferation without interfering with apoptosis. In another approach, Protandim, a combination of 5 well-studied medicinal plants, was given via dietary administration and significantly decreased tumor incidence and multiplicity by $33 \%$ and $57 \%$, respectively. These studies suggest that alterations in antioxidant response may be a novel approach to chemoprevention. This paper focuses on how regulation of antioxidant expression and activity can be modulated in skin disease and the potential clinical implications of antioxidant-based therapies.
\end{abstract}

\section{Introduction}

Antioxidant enzyme expression is known to decrease with aging, which has been theorized to contribute to age-related diseases. One of the main contributors to disease progression is reactive oxygen species (ROS) generation. ROS is the result of incomplete reduction of oxygen within the electron transport chain. The reactive oxidants of ROS include superoxide anion $\left(\mathrm{O}_{2}^{--}\right)$, singlet oxygen $\left(\mathrm{O}_{2}\right)$, hydrogen peroxide $\left(\mathrm{H}_{2} \mathrm{O}_{2}\right)$, and the hydroxyl radical $(\mathrm{OH})$. Although these molecules can act as signaling molecules, they can also participate in cellular damage, such as lipid peroxidation and DNA damage that trigger altered downstream signaling and apoptotic pathways.

With skin being the largest, most readily exposed organ to the environment, it is imperative that mechanisms of protection against oxidative injury are in place within the skin. The skin consists of various antioxidant enzymes such as glutathione reductase, catalase, and superoxide dismutase. These enzymes are often activated to maintain homeostasis and to minimize the damaging effects of ROS. However, alterations in the expression/activity of these antioxidants increase the susceptibility of skin to ROS-mediated injury that contributes to skin disease. Many studies have shown that antioxidant activity, mainly manganese superoxide dismutase (MnSOD), is reduced in various skin cancers. For example, epidermal SOD activity is decreased in hyperproliferative keratinocytes in squamous cell carcinoma, basal cell epithelioma, as well as benign hyperproliferative keratinocytes in the psoriatic epidermis [1-3]. This paper focuses on the therapeutic potential of exogenous antioxidant inducers, the use of SOD mimetics as a chemopreventive agent, and dietary mechanisms of antioxidant induction in skin carcinogenesis.

\section{MnSOD in Skin Disease}

Psoriasis is a skin disease generally characterized by the incomplete differentiation of epidermal keratinocytes, and infiltration of leukocytes. Once localized within skin tissues, these inflammatory cells release various cytokines and ROS resulting in a high incidence of lipid peroxidation. Interestingly, malondialdehyde, a key marker of lipid peroxidation, 
has been found at increased levels in the plasma and red blood cells of patients with psoriatic skin. In addition, plasma levels of b-carotene and a-tocopherol levels were decreased, along with decreased catalase and glutathione-peroxidase activities in RBC, contributing to the pathogenesis of the disease. Lontz et al. examined the mRNA expression of MnSOD in psoriatic skin tissue and found that MnSOD mRNA was significantly higher in lesional psoriatic skin compared to nonlesional skin tissues [4]. Many postulated that associated induction of MnSOD expression may serve as a protective mechanism of increased survival; however, it was not found to be directly correlated to disease pathogenesis. It is known that cytokines such as TNF-a can induce MnSOD expression, suggesting a direct correlation of increased MnSOD expression and the inflammatory mechanism of psoriasis. On the other hand, the oxidative stress-mediated mechanism of disease pathogenesis has been observed in other skin disease, as well, such as contact dermatitis [5], acne, and vitiligo, suggesting the need for further investigation of antioxidantbased interventions in various skin diseases. In this quest, MnSOD becomes a potential target, being that it is the first line of defense within skin tissues and when compared to other SODs such as $\mathrm{Cu}, \mathrm{Zn}-\mathrm{SOD}$, it is affected differently in various skin abnormalities [6]. Therefore, we will continue to further observe the induction, the role of overexpression and chemopreventive potential of MnSOD in other skin diseases, particularly skin carcinogenesis.

\section{Induction of MnSOD Expression}

MnSOD can be induced by a variety of stimuli including cytokines $[7,8]$, radiation $[9,10]$, and chemical carcinogens such as 12-O-tetradecanoylphorbol-3-acetate (TPA). Several studies have shown that TPA can induce MnSOD expression; by direct activation of protein kinase C (PKC) or through the production reactive oxygen species that can act as cell signaling molecules activating redox-sensitive transcription factors such as AP- 1 and NF- $\kappa$ B [11-13]. However, several studies have shown that MnSOD expression is reduced in most human cancers. It has been found that the reduction in MnSOD expression is not due to defects in the primary structure of the MnSOD protein, but rather changes in gene expression $[14,15]$. Several transcription factors have been implicated in the induction of MnSOD expression; however, the most widely studied is specificity protein 1 (Sp1). The transcription factor $S p 1$ contains three zinc finger motifs in the DNA-binding domains that recognize GC-rich sequences of GGGCGG [16]. The GC-rich characteristics of the MnSOD promoter are conserved among mouse [17], bovine [18], rat [19], and human [20]. The Sp1 protein is capable of inducing gene expression by forming homotypic, Sp1-Sp1 interactions [21-23]. However, SP-1 binding affinity and transcription properties can be altered by interactions with other cofactors. Sp1 forms heterotypic interactions with different classes of nuclear proteins such as TATA boxbinding protein (TBP) [24], C/EBP [25], and YY1 [26, 27]. SP-1 recognition sequences are often found to be near binding sites for othertranscription factors such as AP-1
[28], AP-2 [29], and even NF- $\kappa$ B [30] suggesting that SP1 may work in conjunction with other transcription factors to modulate MnSOD gene expression. On the other hand, studies have shown that subcellular organelles, such as the mitochondria can regulate the induction of antioxidant genes such as sod2. Kim et al. showed that in an effort to maintain an optimal mitochondrial redox state, increased MnSOD expression led to endogenous sod2 transcripts, and increased sod2 mRNA levels were a result of increased transcriptional activation of the sod2 gene in mice [31]. In addition, it is known that homozygous sod2-/- knockout mice exhibit a neonatal lethality phenotype that is not reversed or delayed by copper/zinc superoxide dismutase or sod1 overexpression [32]. Therefore, maintenance of the cellular redox state and induction of MnSOD expression is important to cell viability.

\section{Mechanisms of Action of MnSOD}

Initially, it was postulated that flavones and antioxidants inhibit skin carcinogenesis by interfering with the metabolism of carcinogens into the ultimate carcinogen form $[33,34]$. Several studies have found that overexpression as well as deficiencies in MnSOD expression can have significant effects on tumor formation using this two-stage skin carcinogenesis model. Skin carcinogenesis is known to occur in a two-stage process. The two-stage skin carcinogenesis model is a well-established model utilized to study the multiple stages of skin carcinogenesis: tumor initiation, promotion, and metastasis. A single application of the polycyclic aromatic hydrocarbon, 7, 12-dimethylbenz[a] anthracene, is applied at a subthreshold dose. Chemical mutagens, such as DMBA are known to induce carcinogen-specific mutations in the $\mathrm{H}$-ras gene at codon $61[35,36]$. Mutations in the $\mathrm{H}$ ras gene confer a selective advantage within $\mathrm{H}$-ras initiated cells, which can develop into benign tumors after treatment with tumor-promoting agents such as TPA [37]. Following this subcarcinogenic dose, multiple applications of TPA are applied to induce epigenetic changes. The tumor promotion stage is essentially reversible; however, later in the tumorigenesis process, this stage becomes irreversible. Overall, tumor promotion inhibitors have common mechanisms of action: (1) altered metabolism of the carcinogen, (2) scavenging abilities of active molecular species of carcinogens, and lastly (3) competitive inhibition [38]. Several studies have shown the inverse relationship of ROS and MnSOD expression in the pathogenesis of hyperproliferative and inflammatory diseases. It is known that DMBA/TPA treatment induces cell proliferation and apoptosis; both believed to be modulated by oxidative stress propagation [39]. For example, it was found that overexpression of MnSOD, in the two-stage skin carcinogenesis mouse model, reduced the number and incidence of papillomas providing direct evidence of free radical involvement in skin carcinogenesis [40]. Zhao et al. showed that apoptosis preceded cell proliferation [39]. It was found that apoptosis peaked at the 6-hour time point, prior to the peak in cell proliferation at $24 \mathrm{~h}$ [39]. Providing a therapeutic window for antioxidant intervention, 
MnTE-2-PyP ${ }^{5+}$, a small molecule catalytic antioxidant (SOD mimetic), was applied following the peak in apoptosis. It was found that papilloma formation decreased 6-fold compared to their control counterparts, without effecting apoptosis. These results suggest that antioxidant therapy is an effective mode of tumor suppression and can potential be used in conjunction with traditional chemotherapeutics without interfering with drug-induced cell death. Consistent with that, it was found that in the presence of MnTE-2-Pyp ${ }^{5+}$, the level of oxidative injury was significantly reduced. Therefore, these results suggest the oxidative stress-mediated tumor promotion of TPA, as well as, the antioxidant capabilities of MnSOD in tumor suppression. Furthermore, in a study using MnSOD transgenic mice, it was found that only $50 \%$ of transgenic mice developed papillomas, compared to $78 \%$ of their nontransgenic counterparts [40]. These results, again, suggest the antioxidant capabilities of MnSOD as a tumor suppressor.

Moreover, superoxide anions, one of the major constituents of ROS, act as signaling molecules that can regulate oncoproteins and downstream gene expression. As a key cellular redox regulator, MnSOD has been shown to affect the binding activities of transcription factors to transcriptional control elements, therefore modulating gene expression. The mechanism behind MnSOD mediated tumor suppression has been shown to involve suppression of activator protein-1 (AP-1) activity. AP-1 is a key mediator of oncogenic signaling [37]. There are many posttranslational modifications that can regulate AP-1 activity such as modulation of the phosphorylation states of the Jun or Fos protein [41] and redox regulation of the Jun protein. High levels of phosphorylated c-Jun, Fra-1, Fra-2, and ATF-2 proteins have been shown to positively correlate with malignant phenotypes in the multistage mouse skin carcinogenesis model [37]. In addition, the increased expression and posttranslational modifications of these oncoproteins account for a high percentage of the increased AP-1 activity. In malignant cell lines, the DNA binding and transactivation properties of AP1 have been found to be elevated, peaking in fully metastatic cell lines [37]. The transcription factor, AP-1, is known to play a role in cellular differentiation, proliferation, and transformation. The AP-1 complex is known to consist of the homo- or heterodimer of the Fos, Jun, and Fra family members. Many of the subunits of AP-1 are redox sensitive and can be regulated by posttranslational modifications induced by TPA-mediated ROS signaling. It is known that AP-1 activation can be detected as soon as 6 hours postTPA treatment. Zhao et al. showed that by overexpressing MnSOD in human MnSOD transgenic mice, the initial activation of AP-1 was delayed and resulted in a significant reduction in papilloma formation [40]. When both nontransgenic and MnSOD transgenic mice were treated with DMBA/TPA, it was shown that JunD was the only family member whose expression was increased within $24 \mathrm{~h}$ of TPA treatment [40]. Another kinase found to be involved in AP1 activity is c-Jun $\mathrm{N}$-terminal kinase (JNK). JNK activity has been shown to increase more than threefold in malignant cell lines [37]. It was found that the increased phosphorylated form of JNK seen at $6 \mathrm{~h}$ post-TPA treatment in nontransgenic mice was delayed and reduced in MnSOD transgenic mice $24 \mathrm{~h}$ post TPA treatment. These results therefore suggest that MnSOD overexpression can affect TPA-induced AP-1 activation by modulating JNK kinase activity. Nevertheless, we have shown that the induction of endogenous antioxidant enzymes, particularly MnSOD, is efficient in reducing tumor incidence, as well as, mediators of proliferation [40].

\section{Overexpression of MnSOD}

As mentioned previously, overexpression of MnSOD has been shown to be anticarcinogenic in the two-stage skin carcinogenesis model. Overexpression of MnSOD not only reduced tumor multiplicity and incidence, but also modulated cell proliferative pathways such as AP-1signaling and DNA binding activity. In a large number of in vitro and in vivo models, and in even in gene-radiotherapy, MnSOD overexpression has been shown to suppress the malignant phenotype and metastatic ability of tumor cells. MnSOD is an attractive therapeutic target because of its high inducibility and subcellular mitochondrial localization. While various physiological stimuli have been shown to induce MnSOD expression such as cytokines, oxidative stress, and growth factors, the main function of MnSOD is to protect mitochondrial DNA from oxidative injury. Mitochondria are known as the powerhouse of the cell. Not only is mitochondria one of the main energy-generating organelles of the cell, but it is also considered one of the main generators of ROS. Oxygen radicals cannot only act as signaling molecules, but can also promote cell death, which is mainly mediated through the mitochondria. It is known that ROS can amplify the apoptotic cascade by expediting the release of mitochondrial cytochrome $c$ via mitochondrial oxidative damage [42]. With MnSOD being an effective regulator of cellular redox status, this endogenous antioxidant enzyme can also provide cytoprotection from ROS-mediated apoptosis. However, the complexity of MnSOD expression and its involvement cancer progression still remains elusive. Zhao et al., in 2002, showed that a deficiency in MnSOD expression in MnSOD heterozygous knockout (MnSOD KO) mice enhanced cell proliferative signaling [42]. As previously mentioned, AP-1 signaling was suppressed via MnSOD overexpression. Surprisingly, the number of apoptotic cells increased as well, suggesting that MnSOD expression may not only play a role in tumor suppression, but may contribute to cell survival. MnSOD expression has been shown to be increased in various malignancies including human cervical carcinoma [43], brain malignant tumors [44], lung [45], gastric and colon cancers [46]. Consistent with that, in vitro experiments have shown that overexpression of MnSOD protects cells from ionizing radiation and in some cases induces resistance to chemotherapeutic drugs such as adriamycin [47]. Many investigators suggest that the survival mechanisms of MnSOD are mediated by hydrogen peroxide $\left(\mathrm{H}_{2} \mathrm{O}_{2}\right)$ generation that overwhelms the cell capacity to regulate $\mathrm{H}_{2} \mathrm{O}_{2}$ accumulation, promoting cell survival and proliferative signaling. Nevertheless, further studies are needed to elucidate the $\mathrm{H}_{2} \mathrm{O}_{2}$-mediated mechanism. 


\section{MnSOD in Disease: Skin Cancer}

Enzymatic inactivation is known to be associated with most pathological states of disease. With various mechanisms of inactivation, determining the mechanism of inactivation can be complex. Consistent with that, determining the benefits/damaging contributions of MnSOD is controversial, particularly in diseases such as skin carcinogenesis. Therefore, further investigation of activity/expression modulation in various disease states is needed to identify potential therapeutic targets. Previous studies from our lab have shown that ROS generation is increased in the early stages of skin carcinogenesis. It was found that NADPH oxidase was a key contributor to oxidative stress propagation in the DMBA/TPA two-stage skin carcinogenesis model. However, further studies showed that oxidative stress propagation induced p53 mitochondrial translocation. In our in vitro studies, skin epidermal JB6 P+ cells were treated with TPA, 10 minutes post-TPA treatment, the tumor suppressor p53, monitored by immunofluorescence staining, rapidly translocated to the mitochondria. Utilizing immunogold labeling, p53 was found localized on the outer membrane, and surprisingly in the mitochondrial matrix. With both MnSOD and p53 mitochondrial localization being key elements in cell fate, it was found via immunoprecipitation that mitochondrial p53 interacts with MnSOD within the mitochondria. Currently, there is growing controversy surrounding MnSOD's involvement in disease and most importantly the state of MnSOD expression, as well as its activity. Interestingly, following the p53-MnSOD interaction, MnSOD protein levels increased by $60 \%$, whereas its activity decreased $11 \%$, suggesting that MnSOD activity levels may play a more significant role in disease rather than expression. Subsequently following the reduction in MnSOD activity, the transcriptional activity of nuclear p53 was increased 1hour post-TPA treatment, represented by an increase in the proapoptotic protein Bax, a transcriptional target of $\mathrm{p} 53$. In addition, the increase in p53 activation was associated with an increase in DNA fragmentation and apoptotic cell death. However, when treated with the MnSOD mimetic, MnTE$\mathrm{PyP}^{5+}$, mitochondrial p53 levels were slightly reduced, and p53 nuclear translocation and transactivation was completely blocked. Previous studies showed that TPA induced both cell proliferation and p53-mediated apoptosis. However, the involvement of MnSOD modulation in this process remained unsolved. The results from this study provide a link between mitochondrial redox status and nuclear regulation of apoptotic signaling and cell survival.

\section{MnSOD As a Chemopreventive Agent}

MnSOD is a highly inducible enzyme important to cell viability, but can also modulate cell proliferation and apoptotic signaling. Our lab utilized these mechanisms of action as a chemopreventive modality in skin carcinogenesis. Protandim, a dietary supplement consisting of 5 wellestablished medicinal plant extracts, has received increasing attention for itstherapeutic effects in various disease pathologies [47-49]. Protandim consists of B. monnieri (45\% bacosides), $150 \mathrm{mg}$; S. marianum (70-80\% silymarin), $225 \mathrm{mg} ; \quad W$. somnifera (1.5\% withanolides), $150 \mathrm{mg} ; C$. sinensis (98\% polyphenols and 45\% (-)-epigallocatechin3-gallate), $75 \mathrm{mg}$. [50]. Antioxidants have known anticancer effects. However, several large clinical trials using small molecule antioxidants have failed, which poses several questions: Do antioxidant-based therapies contribute to disease progression by becoming prooxidants themselves? Are antioxidant-based therapies potent enough to overcome the ROS-generating load of various disease states? In general, cancer cells have a higher ROS generation status than normal cells. As a result, a number of antioxidant enzymes are significantly reduced in expression levels and activity. In addition, polymorphisms in MnSOD have been shown to be associated with a higher risk of prostate, breast, and other various cancers. However, MnSOD is the only antioxidant enzyme shown, when overexpressed, to reduce multicancer cell growth both in vitro and in vivo [51]. In other studies, small molecule catalytic antioxidant enzymes have been shown to be a more potent and practical approach for cancer chemoprevention. Sporn and Suh define chemoprevention as a pharmacological approach to intervention in order to arrest or reverse the process of carcinogenesis [52]. In our study, the two-stage mouse skin carcinogenesis model was used to investigate the mechanisms of action of Protandim during the early stages of skin carcinogenesis. Overall, the process of carcinogenesis is mediated by ROS generation and the ability of oxygen radicals to act as signaling molecules to modulate downstream carcinogenic events. We have found that the positive feedback loop that is formed by oxidative stress, cell proliferation and p53-mediated apoptosis plays a major role in contributing to carcinogenesis. Thus, it was postulated that the induction of MnSOD via Protandim could break this positive feedback cycle leading to cancer prevention. Mice utilized in this study were fed the Protandim diet during the tumor promotion stage (i.e., 2 weeks following DMBA initiation and 2 weeks prior to TPA treatment and for the duration of the study). Overall, no tumors were formed in the vehicle control/basal diet groups. However, both tumor incidence and multiplicity were reduced by $33 \%$ and 57\%, respectively, in the Protandim diet group [53]. These results suggest that modulation of oxidative stress through the induction of antioxidant enzymes via dietary administration is sufficient in reducing tumor formation.

Nonetheless, oxidative stress alters both gene expression and cancer biology. Another key component of tumor progression is inflammation. Within the tumor microenvironment, various inflammatory cells release ROS and other inflammatory mediators. Tumors often utilize these pro-inflammatory mediators to foster cell proliferation, angiogenesis and metastasis [54]. Utilizing the two-stage model, we found that dietary administration of Protandim significantly decreased TPA-mediated macrophage infiltration, as well as, pro-inflammatory signaling pathways. For example, nuclear factor kappa B $(\mathrm{NF}-\kappa \mathrm{B})$, a central regulator of immunity and inflammation, is a transcription factor of biological interest because of its sensitivity to the intracellular redox status. NF- $\kappa \mathrm{B}$ regulates the expression 
of numerous genes that encode selectins, cytokines, and cellular adhesion molecules. Oxidative stress generation is known to induce NF- $\kappa$ B nuclear translocation. Intracellular adhesion molecule-1 (ICAM-1) and vascular cell adhesion molecule-1 (VCAM-1) are transcriptionally regulated by NF- $\kappa$ B. Skin tissues from mice fed the Protandim diet exhibited reduced NF- $\kappa$ B DNA binding activity, resulting in a reduction in the protein expression levels of both cellular adhesion molecules. Therefore, these results suggest that Protandim not only suppresses tumor formation, but also mechanistically modulates pro-inflammatory signaling and the immune response via gene transcription.

As mentioned previously, p53 interacts with MnSOD following TPA-mediated oxidative stress generation. Therefore, is it possible for a dietary-mediated induction of MnSOD expression/activity to modulate p53 mitochondrial translocation and accompanying apoptosis? Skin tissues from DMBA/TPA treated mice were analyzed to assess the effects of the Protandim diet on p53 mitochondrial translocation [55]. Interestingly, skin tissues from Protandim-fed mice showed a significant decrease in mitochondrial p53 protein expression. Consistent with that, the number of apoptotic cells was also significantly decreased. Thus, the induction of antioxidant enzymes via dietary administration of Protandim modulates both TPA-mediated cell proliferation and p53-mediated apoptotic signaling. Therefore, it can be concluded that oxidative stress forms a mechanistic linkage between cell proliferation, inflammation, and apoptosis, suggesting that potent multimodal antioxidant inducers may potentially be utilized with conventional chemotherapeutics.

\section{Conclusion}

For many decades, ROS generation has been known to not only cause oxidative injury, but also act as signaling molecules that regulate cell proliferation and downstream gene expression. However, the induction of MnSOD is gaining interest as an effective novel mechanism of chemoprevention, being that it is the only antioxidant enzyme that when overexpressed suppresses tumor formation. MnSOD also has the ability to modulate multiple pathways contributing to skin carcinogenesis. Continuous efforts are currently being made to develop compounds that effectively induce MnSOD in hopes to incorporate antioxidant-based therapies into current clinical practice. Therefore, the development of various MnSOD inducers to be used during the early-onset of tumorigenesis may be a plausible modality utilized to suppress underlying mechanisms involved in carcinogenesis.

\section{References}

[1] N. Ohkuma, S. Kajita, and H. Iizuka, "Superoxide dismutase in epidermis: its relation to keratinocyte proliferation," Journal of Dermatology, vol. 14, no. 6, pp. 562-568, 1987.

[2] T. Galeotti, S. Borrello, and A. Seccia, "Superoxide dismutase content in human epidermis and squamous cell epithelioma," Archives of Dermatological Research, vol. 267, no. 1, pp. 83-86, 1980.
[3] H. M. van Baar, P. C. van de Kerkhof, J. Schalkwijk, and P. D. Mier, "Cutaneous superoxide dismutase activity in psoriasis," British Journal of Dermatology, vol. 116, no. 3, pp. 462-463, 1987.

[4] W. Lontz, A. Sirsjo, W. Liu, M. Lindberg, O. Rollman, and H. Torma, "Increased mRNA expression of manganese superoxide dismutase in psoriasis skin lesions and in cultured human keratinocytes exposed to IL- $1 \beta$ and TNF- $\alpha$," Free Radical Biology and Medicine, vol. 18, no. 2, pp. 349-355, 1995.

[5] J. Fuchs, T. M. Zollner, R. Kaufmann, and M. Podda, "Redoxmodulated pathways in inflammatory skin diseases," Free Radical Biology and Medicine, vol. 30, no. 4, pp. 337-353, 2001.

[6] T. Kobayashi, M. Matsumoto, H. Iizuka, K. Suzuki, and N. Taniguchi, "Superoxide dismutase in psoriasis, squamous cell carcinoma and basal cell epithelioma: an immunohistochemical study," British Journal of Dermatology, vol. 124, no. 6, pp. 555-559, 1991.

[7] G. H. W. Wong and D. V. Goeddel, "Induction of manganous superoxide dismutase by tumor necrosis factor: possible protective mechanism," Science, vol. 242, no. 4880, pp. 941944, 1988.

[8] L. A. H. Borg, E. Cagliero, S. Sandler, N. Welsh, and D. L. Eizirik, "Interleukin-1 $\beta$ increases the activity of superoxide dismutase in rat pancreatic islets," Endocrinology, vol. 130, no. 5, pp. 2851-2857, 1992.

[9] L. W. Oberley, D. K. St. Clair D.K., A. P. Autor, and T. D. Oberley, "Increase in manganese superoxide dismutase activity in the mouse heart after X-irradiation," Archives of Biochemistry and Biophysics, vol. 254, no. 1, pp. 69-80, 1987.

[10] M. Akashi, M. Hachiya, R. L. Paquette, Y. Osawa, S. Shimizu, and G. Suzuki, "Irradiation increases manganese superoxide dismutase mRNA levels in human fibroblasts. Possible mechanisms for its accumulation," Journal of Biological Chemistry, vol. 270, no. 26, pp. 15864-15869, 1995.

[11] P. Angel, I. Baumann, B. Stein, H. Delius, H. J. Rahmsdorf, and P. Herrlich, "12-O-tetradecanoyl-phorbol-13-acetate induction of the human collagenase gene is mediated by an inducible enhancer element located in the 5'-flanking region," Molecular and Cellular Biology, vol. 7, no. 6, pp. 2256-2266, 1987.

[12] M. R. Edbrooke, D. W. Burt, J. K. Cheshire, and P. Woo, "Identification of cis-acting sequences responsible for phorbol ester induction of human serum amyloid A gene expression via a nuclear factor $\kappa \mathrm{B}$-like transcription factor," Molecular and Cellular Biology, vol. 9, no. 5, pp. 1908-1916, 1989.

[13] J. Fujii and N. Taniguchi, "Phorbol ester induces manganesesuperoxide dismutase in tumor necrosis factor-resistant cells," Journal of Biological Chemistry, vol. 266, no. 34, pp. 2314223146, 1991.

[14] Y. Xu, A. Krishnan, X. S. Wan et al., "Mutations in the promoter reveal a cause for the reduced expression of the human manganese superoxide dismutase gene in cancer cells," Oncogene, vol. 18, no. 1, pp. 93-102, 1999.

[15] D. K. St. Clair D.K. and J. C. Holland, "Complementary DNA encoding human colon cancer manganese superoxide dismutase and the expression of its gene in human cells," Cancer Research, vol. 51, no. 3, pp. 939-943, 1991.

[16] W. S. Dynan and R. Tjian, "The promoter-specific transcription factor Sp 1 binds to upstream sequences in the SV40 early promoter," Cell, vol. 35, no. 1, pp. 79-87, 1983.

[17] P. L. Jones, G. Kucera, H. Gordon, and J. M. Boss, "Cloning and characterization of the murine manganous superoxide dismutase-encoding gene," Gene, vol. 153, no. 2, pp. 155-161, 1995. 
[18] B. Meyrick and M. A. Magnuson, "Identification and functional characterization of the bovine manganous superoxide dismutase promoter," American Journal of Respiratory Cell and Molecular Biology, vol. 10, no. 1, pp. 113-121, 1994.

[19] Y. S. Ho, A. J. Howard, and J. D. Crapo, "Molecular structure of a functional rat gene for manganese-containing superoxide dismutase," American journal of respiratory cell and molecular biology, vol. 4, no. 3, pp. 278-286, 1991.

[20] X. S. Wan, M. N. Devalaraja, and D. K. ST. Clair, "Molecular structure and organization of the human manganese superoxide dismutase gene," DNA and Cell Biology, vol. 13, no. 11, pp. 1127-1136, 1994.

[21] I. A. Mastrangelo, A. J. Courey, J. S. Wall, S. P. Jackson, and P. V. C. Hough, "DNA looping and Sp1 multimer links: a mechanism for transcriptional synergism and enhancement," Proceedings of the National Academy of Sciences of the United States of America, vol. 88, no. 13, pp. 5670-5674, 1991.

[22] E. Pascal and R. Tjian, "Different activation domains of Sp1 govern formation of multimers and mediate transcriptional synergism," Genes and Development, vol. 5, no. 9, pp. 16461656, 1991.

[23] W. Su, S. Jackson, R. Tjian, and H. Echols, "DNA looping between sites for transcriptional activation: self-association of DNA-bound Sp1," Genes and Development, vol. 5, no. 5, pp. 820-826, 1991.

[24] A. Emili, J. Greenblatt, and C. J. Ingles, "Species-specific interaction of the glutamine-rich activation domains of $\mathrm{Sp} 1$ with the TATA box-binding protein," Molecular and Cellular Biology, vol. 14, no. 3, pp. 1582-1593, 1994.

[25] Y. H. Lee, S. C. Williams, M. Baer, E. Sterneck, F. J. Gonzalez, and P. F. Johnson, "The ability of $\mathrm{C} / \mathrm{EBP} \beta$ but not $\mathrm{C} / \mathrm{EBP} \alpha$ to synergize with an Sp1 protein is specified by the leucine zipper and activation domain," Molecular and Cellular Biology, vol. 17, no. 4, pp. 2038-2047, 1997.

[26] J. S. Lee, K. M. Galvin, and Y. Shi, "Evidence for physical interaction between the zinc-finger transcription factors YY1 and Sp1," Proceedings of the National Academy of Sciences of the United States of America, vol. 90, no. 13, pp. 6145-6149, 1993.

[27] E. Seto, B. Lewis, and T. Shenk, "Interaction between transcription factors Sp1 and YY1," Nature, vol. 365, no. 6445, pp. 462-464, 1993.

[28] W. Lee, A. Haslinger, M. Karin, and R. Tjian, "Activation of transcription by two factors that bind promoter and enhancer sequences of the human metallothionein gene and SV40," Nature, vol. 325, no. 6102, pp. 368-372, 1987.

[29] D. K. Getman, A. Mutero, K. Inoue, and P. Taylor, “Transcription factor repression and activation of the human acetylcholinesterase gene," Journal of Biological Chemistry, vol. 270, no. 40, pp. 23511-23519, 1995.

[30] N. D. Perkins, A. B. Agranoff, E. Pascal, and G. J. Nabel, “An interaction between the DNA-binding domains of RelA(p65) and Sp1 mediates human immunodeficiency virus gene activation," Molecular and Cellular Biology, vol. 14, no. 10, pp. 6570-6583, 1994.

[31] A. Kim, M. P. Murphy, and T. D. Oberley, "Mitochondrial redox state regulates transcription of the nuclear-encoded mitochondrial protein manganese superoxide dismutase: a proposed adaptive response to mitochondrial redox imbalance," Free Radical Biology and Medicine, vol. 38, no. 5, pp. 644-654, 2005.
[32] J. C. Copin, Y. Gasche, and P. H. Chan, "Overexpression of copper/zinc superoxide dismutase does not prevent neonatal lethality in mutant mice that lack manganese superoxide dismutase," Free Radical Biology and Medicine, vol. 28, no. 10, pp. 1571-1576, 2000.

[33] D. H. Phillips, P. L. Grover, and P. Sims, "A quantitative determination of the covalent binding of a series of polycyclic hydrocarbons to DNA in mouse skin," International Journal of Cancer, vol. 23, no. 2, pp. 201-208, 1979.

[34] T. J. Slaga and W. M. Bracken, "The effects of antioxidants on skin tumor initiation and aryl hydrocarbon hydroxylase," Cancer Research, vol. 37, no. 6, pp. 1631-1635, 1977.

[35] A. Balmain and K. Brown, "Oncogene activation in chemical carcinogenesis," Advances in Cancer Research, vol. 51, pp. 147$182,1988$.

[36] M. Quintanilla, K. Brown, M. Ramsden, and A. Balmain, "Carcinogen-specific mutation and amplification of Ha-ras during mouse skin carcinogenesis," Nature, vol. 322, no. 6074, pp. 78-80, 1986.

[37] V. Zoumpourlis, P. Papassava, S. Linardopoulos, D. Gillespie, A. Balmain, and A. Pintzas, "High levels of phosphorylated cJun, Fra-1, Fra-2 and ATF-2 proteins correlate with malignant phenotypes in the multistage mouse skin carcinogenesis model," Oncogene, vol. 19, no. 35, pp. 4011-4021, 2000.

[38] T. J. Slaga, "Overview of tumor promotion in animals," Environmental Health Perspectives, vol. 50, pp. 3-14, 1983.

[39] Y. Zhao, Y. Xue, T. D. Oberley et al., "Overexpression of manganese superoxide dismutase suppresses tumor formation by modulation of activator protein-1 signaling in a multistage skin carcinogenesis model," Cancer Research, vol. 61, no. 16, pp. 6082-6088, 2001.

[40] Y. Zhao, L. Chaiswing, T. D. Oberley et al., "A mechanismbased antioxidant approach for the reduction of skin carcinogenesis," Cancer Research, vol. 65, no. 4, pp. 1401-1405, 2005.

[41] W. J. Boyle, T. Smeal, L. H. K. Defize et al., "Activation of protein kinase $\mathrm{C}$ decreases phosphorylation of c-Jun at sites that negatively regulate its DNA-binding activity," Cell, vol. 64, no. 3, pp. 573-584, 1991.

[42] Y. Zhao, T. D. Oberley, L. Chaiswing et al., "Manganese superoxide dismutase deficiency enhances cell turnover via tumor promoter-induced alterations in AP-1 and p53-mediated pathways in a skin cancer model," Oncogene, vol. 21, no. 24, pp. 3836-3846, 2002.

[43] M. Landriscina, F. Remiddi, F. Ria et al., "The level of MnSOD is directly correlated with grade of brain tumours of neuroepithelial origin," British Journal of Cancer, vol. 74, no. 12, pp. 1877-1885, 1996.

[44] J. C. M. Ho, S. Zheng, S. A. A. Comhair, C. Farver, and S. C. Erzurum, "Differential expression of manganese superoxide dismutase and catalase in lung cancer," Cancer Research, vol. 61, no. 23, pp. 8578-8585, 2001.

[45] A. M. L. Janssen, C. B. Bosman, C. F. M. Sier et al., "Superoxide dismutases in relation to the overall survival of colorectal cancer patients," British Journal of Cancer, vol. 78, no. 8, pp. 1051-1057, 1998.

[46] K. Hirose, D. L. Longo, J. J. Oppenheim, and K. Matsushima, "Overexpression of mitochondrial manganese superoxide dismutase promotes the survival of tumor cells exposed to interleukin-1, tumor necrosis factor, selected anticancer drugs, and ionizing radiation," FASEB Journal, vol. 7, no. 2, pp. 361368, 1993. 
[47] H. J. Bogaard, R. Natarajan, S. C. Henderson et al., "Chronic pulmonary artery pressure elevation is insufficient to explain right heart failure," Circulation, vol. 120, no. 20, pp. 19511960, 2009.

[48] B. Joddar, R. K. Reen, M. S. Firstenberg et al., "Protandim attenuates intimal hyperplasia in human saphenous veins cultured ex vivo via a catalase-dependent pathway," Free Radical Biology and Medicine, vol. 50, no. 6, pp. 700-709, 2011.

[49] M. M. Qureshi, W. C. McClure, N. L. Arevalo et al., "The dietary supplement protandim $(\mathrm{B}$ ) decreases plasma osteopontin and improves markers of oxidative stress in muscular dystrophy Mdx mice," Journal of Dietary Supplements, vol. 7, no. 2, pp. 159-178, 2010.

[50] S. K. Nelson, S. K. Bose, G. K. Grunwald, P. Myhill, and J. M. McCord, "The induction of human superoxide dismutase and catalase in vivo: a fundamentally new approach to antioxidant therapy," Free Radical Biology and Medicine, vol. 40, no. 2, pp. 341-347, 2006.

[51] Y. Zhao, L. Chaiswing, J. M. Velez et al., "p53 translocation to mitochondria precedes its nuclear translocation and targets mitochondrial oxidative defense protein-manganese superoxide dismutase," Cancer Research, vol. 65, no. 9, pp. 3745-3750, 2005.

[52] M. B. Sporn and N. Suh, "Chemoprevention of cancer," Carcinogenesis, vol. 21, no. 3, pp. 525-530, 2000.

[53] J. Liu, X. Gu, D. Robbins et al., "Protandim, a fundamentally new antioxidant approach in chemoprevention using mouse two-stage skin carcinogenesis as a model," PloS ONE, vol. 4, no. 4, Article ID e5284, 2009.

[54] L. M. Coussens and Z. Werb, "Inflammation and cancer," Nature, vol. 420, no. 6917, pp. 860-867, 2002.

[55] D. Robbins, X. Gu, R. Shi et al., "The chemopreventive effects of Protandim: modulation of p53 mitochondrial translocation and apoptosis during skin carcinogenesis," PLOS ONE, vol. 5, no. 7, Article ID e11902, 2010. 

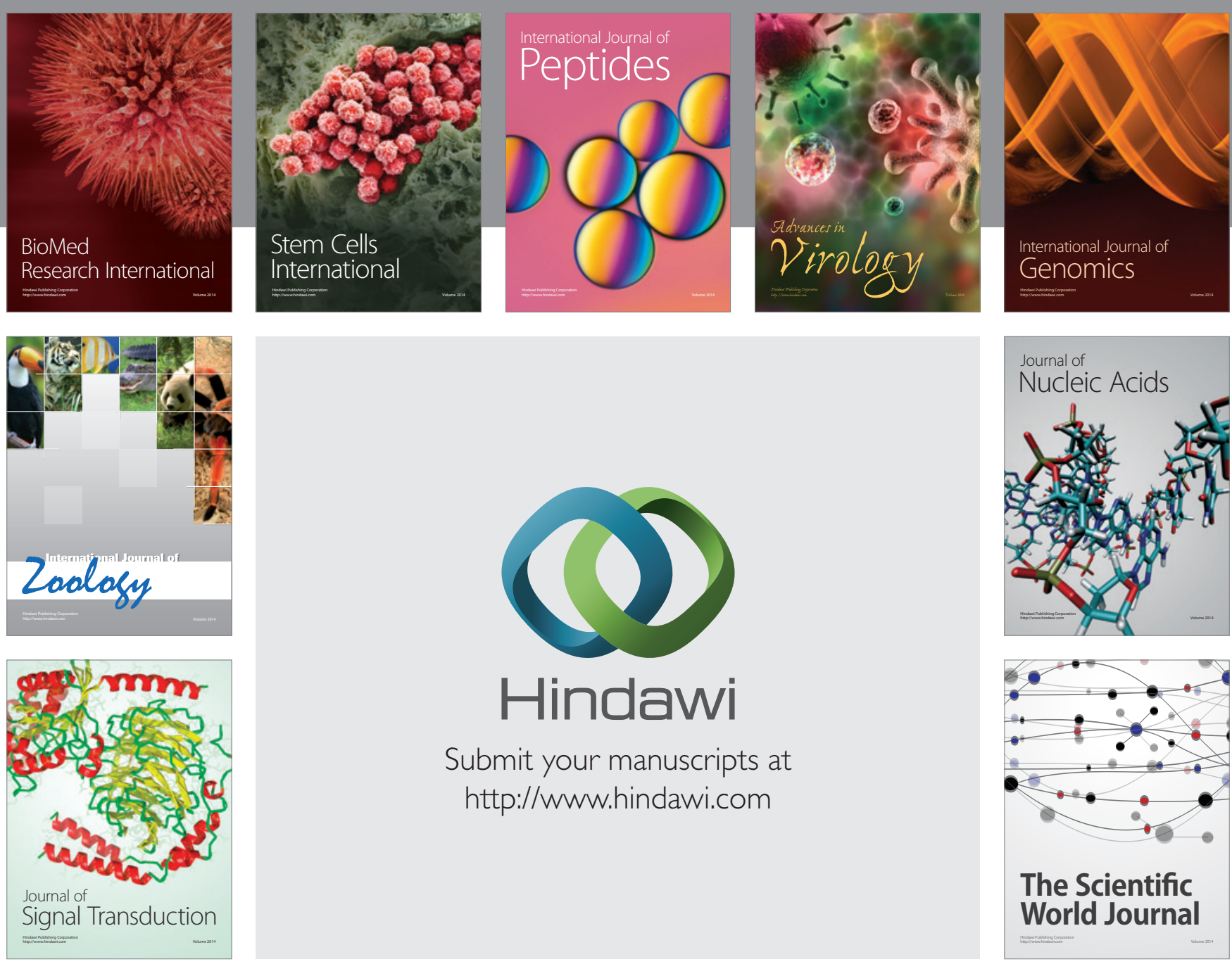

Submit your manuscripts at

http://www.hindawi.com
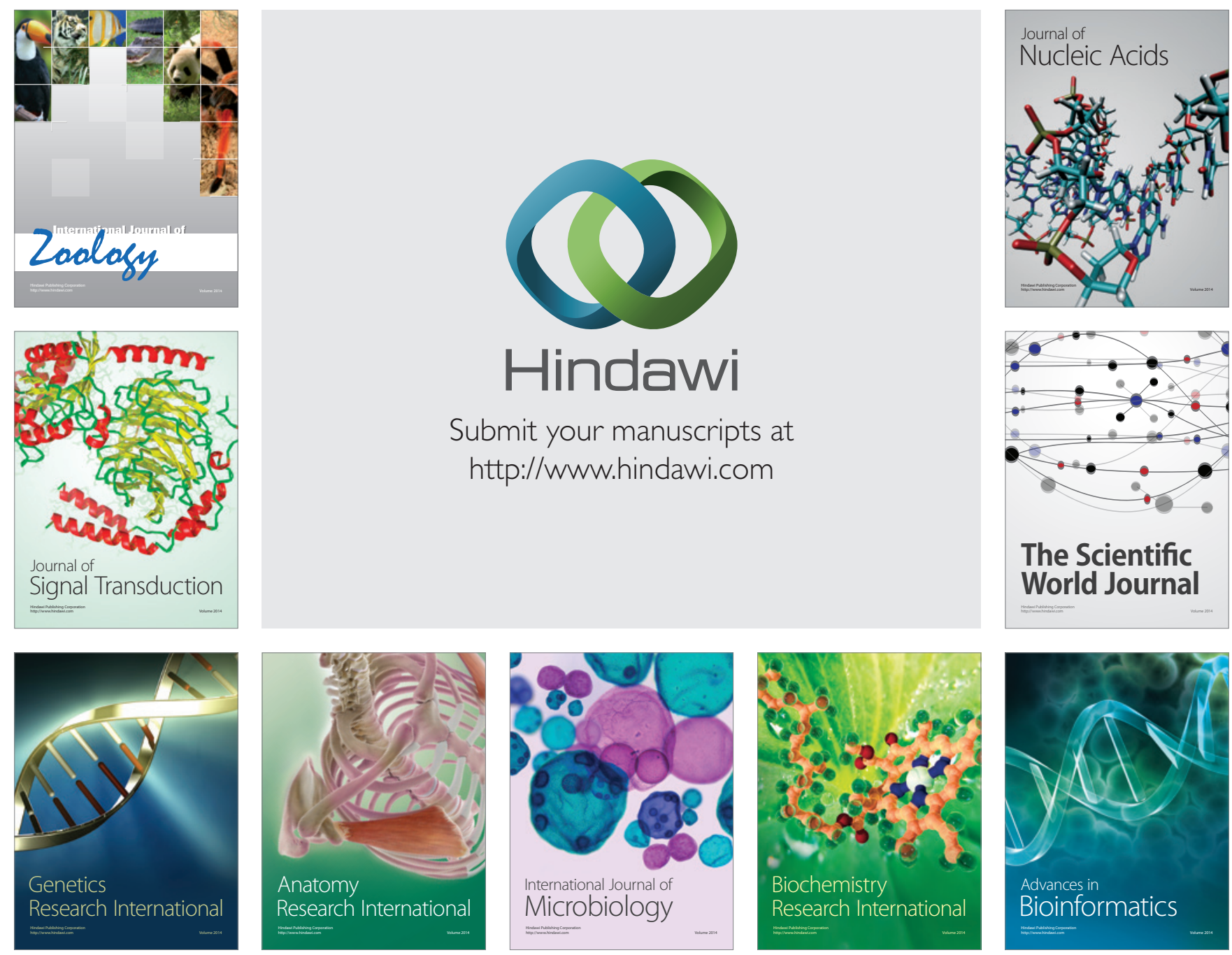

The Scientific World Journal
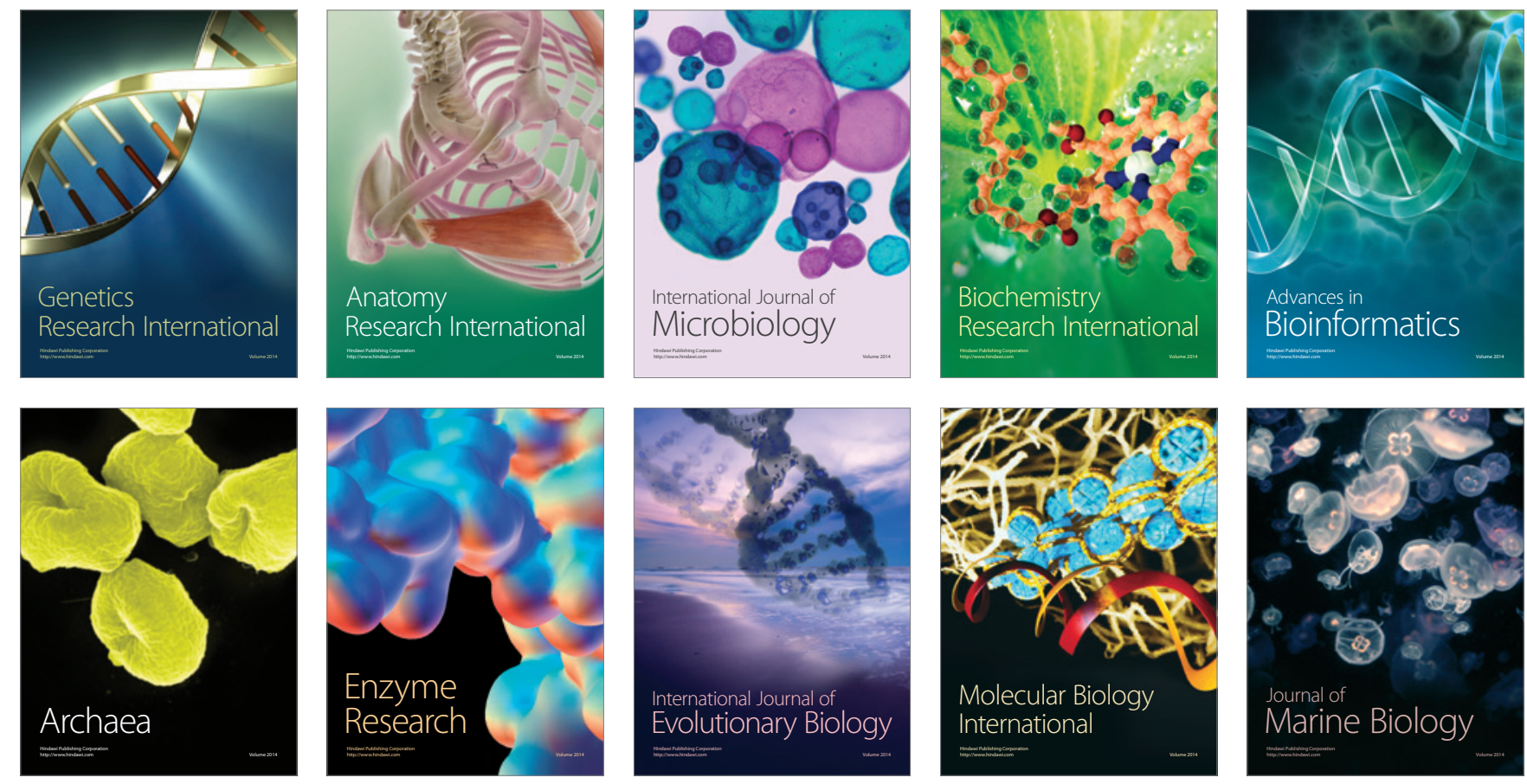